\title{
A METHOD FOR THE ISOLATION OF BACTEROIDES MELANINOGENICUS FROM THE HUMAN MOUTH
}

\author{
W. P. Holbrook* $\ddagger$, S. A. Ogston† ANd P. W. Ross* \\ * Department of Bacteriology, University Medical School, Teviot Place, \\ Edinburgh EH8 $9 A G$, and \\ $\dagger$ Department of Statistics, The King's Buildings, University of Edinburgh, \\ Edinburgh EH9 $3 \mathrm{JZ}$
}

SEveral elaborate procedures for the isolation of anaerobes from the gingival sulcus have been described. These include the use of oxygen-free gas to protect the specimen during sampling (Berg and Nord, 1973) and a portable, positive-pressure, low-oxygen box for the collection of samples (Sutter et al., 1972). These methods are often not considered feasible in dental hospitals and other clinical laboratories. Consequently a simple method was sought that would allow reliable isolation of anaerobic bacteria and that would be acceptable as a routine procedure in a dental hospital. Watt, Collee and Brown (1974) have shown that careful bench handling of specimens of faeces yielded anaerobes in numbers similar to those obtained by manipulation in an anaerobic cabinet. Rosenblatt, Fallon and Finegold (1973) and Starr (1974) have also found no advantage in the use of roll tubes over conventional bench methods. The techniques of Collee et al. (1972) and Watt, Hoare and Collee (1973) were adopted in this study.

The amounts of material obtainable from the gingival sulcus, particularly from healthy mouths, make quantitative estimations of bacteria difficult. Specimens weighed in air are subject to desiccation and prolonged exposure to oxygen, while samples held in gassed tubes weigh but a fraction of the weight of the container and consequently the error in weighing is great. Dispersal of the specimen presents further problems. It was therefore decided to study a number of different isolation procedures and to compare their relative merits as methods for isolating Bacteroides melaninogenicus from specimens. This species was considered to be a useful indicator because it is a fairly exacting anaerobe and its black colonies are readily recognisable on blood-containing media. Several selective and nonselective solid media, transport media and liquid media were compared in preliminary experiments on the quantitative and qualitative recovery of standard strains (Holbrook, 1976). In the present experiment several media and methods of isolation were studied in a clinical survey.

\section{MATERIALS AND METHODS}

Anaerobic incubation. All anaerobic incubation was carried out in anaerobic jars (Baird and Tatlock Ltd) fitted with three room-temperature catalyst sachets. The jars were filled with a mixture of $\mathrm{H}_{2} 90 \%$ and $\mathrm{CO}_{2} \quad 10 \%$ (BOC Special Gases) according to the method of Collee et al. (1972).

Solid media. The solid medium was derived from the BM medium of Nash, referred to by Williams et al. (1975); it contained Trypticase (BBL) 1\%, Proteose Peptone (Oxoid) $1 \%$, Yeast Extract (Difco) $0.5 \%$, sodium chloride $0.5 \%$, sodium succinate $0.25 \%$, and Ionagar

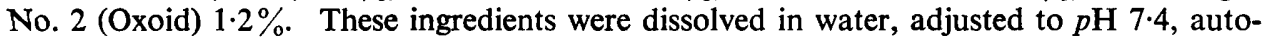
claved at $121^{\circ} \mathrm{C}$ for $15 \mathrm{~min}$. and allowed to cool to $50^{\circ} \mathrm{C}$. Saponin-lysed human blood $5 \%$ ( $\mathrm{v} / \mathrm{v})$, menadione $1 \mu \mathrm{g}$ per $\mathrm{ml}$, and L-cysteine hydrochloride $0.075 \%$ were added to the cooled medium and the plates poured.

Received 5 Oct. 1977; accepted 28 Oct. 1977.

$\ddagger$ Present Address: Department of Oral Medicine, Turner Dental School, Bridgeford Street, Manchester M15 6FH.

J. MED. MICROBIOL.-VOL. 11 (1978) 
Two selective media were prepared by adding to the cooled medium either kanamycin $75 \mu \mathrm{g}$ per ml or kanamycin $75 \mu \mathrm{g}$ per ml plus vancomycin $2.5 \mu \mathrm{g}$ per ml (Finegold, Sugihara and Sutter, 1971). Solid media were prepared the day before use and stored overnight anaerobically at room temperature in jars that were opened immediately before use.

Enrichment broth medium. The ingredients in the basic enrichment broth were the same as those in solid medium except that the Ionagar was omitted. The ingredients were dissolved

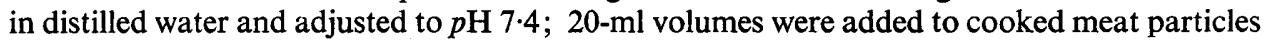
approximately $1 \mathrm{~cm}$ in depth in screw-capped bottles of 28-ml capacity. The medium was autoclaved at $121^{\circ} \mathrm{C}$ for $15 \mathrm{~min}$. and stored at $4^{\circ} \mathrm{C}$. To the medium just before use were added haemin $5 \mu \mathrm{g}$ per $\mathrm{ml}$ and menadione $1 \mu \mathrm{g}$ per $\mathrm{ml}$. Bottles were held anaerobically overnight at $4^{\circ} \mathrm{C}$ and the screw-caps were immediately tightened on removal from the jar.

Transport medium. VMGII fluid-storage medium was prepared as described by Möller (1966) and stored aerobically at room temperature in screw-capped bottles of 7-ml capacity.

Sampling procedure. Samples were collected from 10 patients attending the periodontal clinic of the Edinburgh Dental Hospital and from 40 clinical dental students. The degree of periodontal disease of each subject was scored by Russell's Index as described by MacPhee and Cowley (1975). Wooden swab-sticks cut to produce a chisel-edge were autoclaved and used to collect samples from the gingival sulci of one or more teeth. Five samples were taken from each subject; portions of three were immediately placed on the surface of the non-

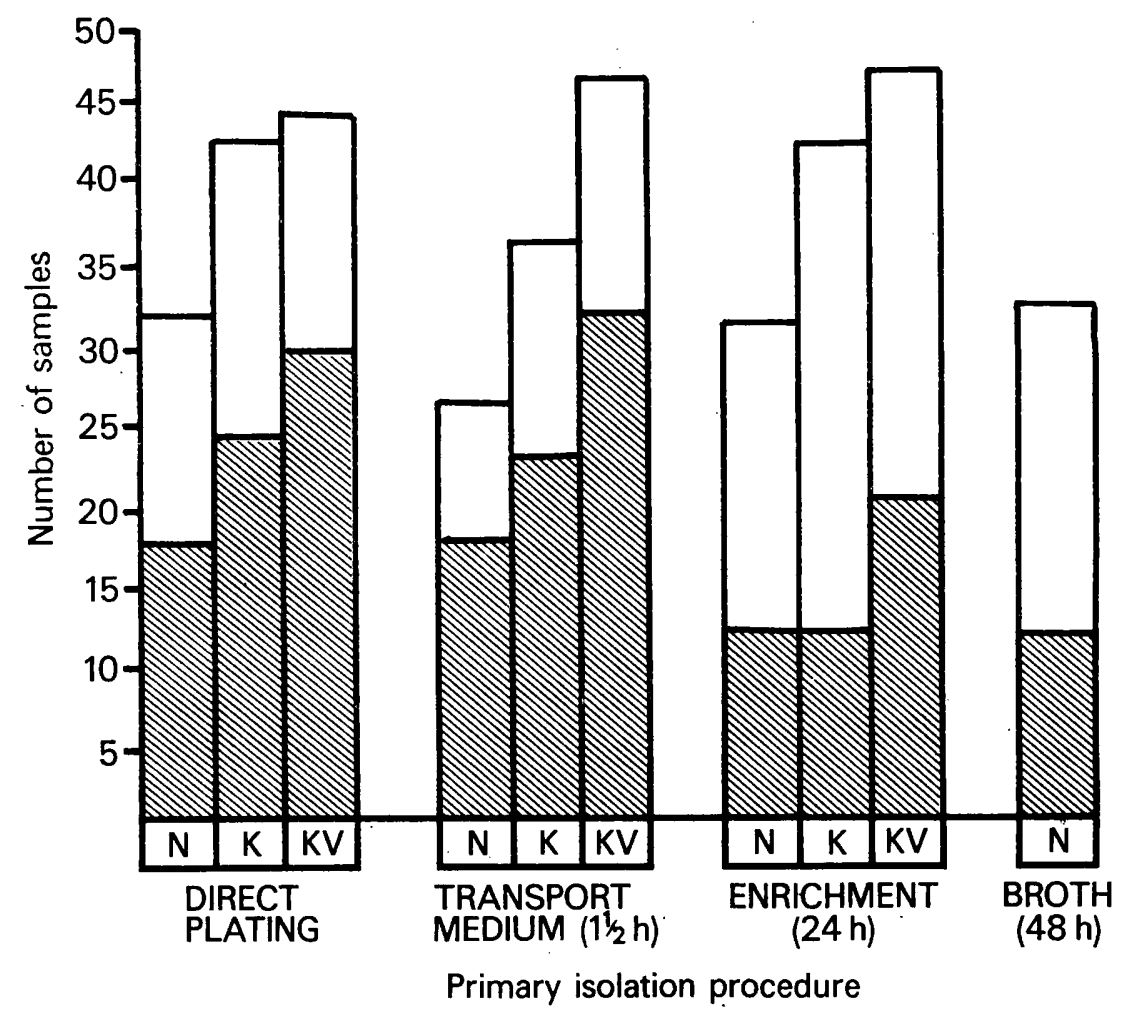

FiguRE.-Bar chart to illustrate the relative merits of 10 different isolation procedures for recovering Bacteroides melaninogenicus from human oral samples. Each bar represents an isolation procedure. $\mathrm{N}=$ Non-selective medium, $\mathrm{K}=$ selective medium containing kanamycin, and $\mathrm{KV}=$ selective medium containing kanamycin and vancomycin. The shaded portion of the bar indicates the number of samples from which B. melaninogenicus was successfully subcultured; the unshaded portion $\square$ shows the number of samples in which organisms capable of yielding black- or brown-pigmented colonies were present but failed to grow on subculture. The total height of the bar thus represents the total number of samples containing such organisms. 
selective medium and the two selective media; a fourth and fifth sample were used to seed enrichment broth and VMGII transport medium, respectively.

Inoculated media were returned promptly to the laboratory where streaking-out of the samples placed directly on solid media was completed. The bottles of VMGII transport medium were placed in an ultrasonic bath (Kerry's Ultrasonics Ltd, Model K/B 80/2) and the samples dispersed by ultrasonication at $80 \mathrm{kHz}$ for $15 \mathrm{~min}$. One loopful $(c .0 \cdot 1 \mathrm{ml})$ from each sample in transport medium was plated on each of the two selective media and on nonselective medium. A similar plating procedure was used for loopfuls from inoculated bottles of enrichment broth after anaerobic incubation for $24 \mathrm{~h}$; after incubation for a further $24 \mathrm{~h} \mathrm{a}$ further loopful from each bottle was seeded on to a plate of non-selective medium. All plates were incubated anaerobically.

Examination of plates. All plates were examined at daily intervals for the growth of any black- or brown-pigmented colonies. Representative colonies were subcultured aerobically and anaerobically on non-selective medium and examined in Gram-stained smears. Blackor brown-pigmented colonies of Gram-negative bacilli that grew only anaerobically were identified as $B$. melaninogenicus. Persistent attempts were made to subculture a black- or brown-pigmented colony from each plate.

\section{RESULTS}

Isolation of B. melaninogenicus from each subject was attempted by 10 different technical procedures (figure), and for each procedure one of three possible results was recorded, namely $(a)$ absence of black or brown colonies, $(b)$ black or brown colonies present that failed to grow in pure subculture, and $(c)$ black or brown colonies present that were successfully grown in pure subculture. The totals for each category of results and for each isolation procedure are shown in the figure. In respect of the black-pigmented colonies that grew, there was little difference between plates inoculated with samples directly and those inoculated from transport medium, but plates inoculated from enrichment broth less frequently yielded black-pigmented colonies in pure culture. The number of samples that yielded a pure growth of black-pigmented colonies on subculture was increased by the use of plates containing kanamycin, and increased still more if they contained both kanamycin and vancomycin.

Results of statistical analysis. The results recorded for each sample ( $a, b$ and $c$ above) were assigned "scores" 1,2 and 3 and the significance of " subject" and "treatment" variations was analysed by a two-way analysis of variance (Snedecor and Cochran, 1967). Although this method assumes an underlying linear model with a normally distributed error, which is obviously unrealistic here, computer simulations showed that it gave reliable results when, as here, a reasonably large number of observations was made (Ogston, 1976). Significant variation was found between subjects. Little difference was detected between the direct plating technique and the use of transport media, but both methods gave significantly better results than did the enrichment broth procedure $(\mathrm{P}<0.01)$. There was no evidence to suggest that any sampling method was better with a particular selective agent.

The effects of the different methods of sample treatment were further analysed in the following two stages: firstly, according to whether or not black-pigmented colonies were present; secondly, whether black colonies, when present, were successfully grown in pure subculture. At each stage results were analysed by means of an extension of McNemar's test (Armitage, 1971).

Chi-square analysis of the results at the first stage did not detect any significant difference between the isolation methods of direct plating, use of transport medium or use of enrichment broth. Addition of kanamycin to the solid medium significantly increased the frequency with which black- or brown-pigmented colonies were demonstrated, and a combination of kanamycin and vancomycin was still more effective. A similar chi-square analysis at the second stage indicated that the enrichment broth gave significantly poorer results for the eventual isolation of pure cultures of B. melaninogenicus. There was evidence that kanamycin plus vancomycin produced a significantly greater number of pure subcultures than either kanamycin alone or the non-selective medium. 
There was no correlation between Russell's Index score and the success of any method of isolating B. melaninogenicus strains in either pure or mixed culture (correlation coefficient $-0 \cdot 13)$.

\section{Discussion}

The results indicated that $B$. melaninogenicus could be isolated, in mixed culture at least, from the gingival sulcus of almost all subjects, even those with comparatively little periodontal disease. Thirty-nine subjects had healthy gingival tissues or gingivitis that had not progressed to an established periodontitis. There was no apparent relationship between the success of isolating $B$. melaninogenicus and the degree of periodontal disease, although numbers were small in the higher disease scores. Sampling and isolation procedures appear to be reliable even when there was little gingival debris.

The failure to demonstrate any significant difference in isolation rates between samples directly plated immediately after collection and those held in transport medium indicated that VMGII may be a useful medium for clinical use. Möller (1966) has demonstrated the value of this transport medium for endodontic specimens. Its long shelf-life and the ease with which it may be stored make it ideal for use in clinical practice; the distribution to clinics of freshly prepared, anaerobically stored plates would be expensive in time and materials.

Irrespective of the isolation procedure adopted, it was clear that a number of organisms capable of yielding black-pigmented colonies were present but failed to grow on subculture. These may have belonged to particularly exacting strains dependent on nutrients from other bacteria in the mixed culture. In related studies some strains of $B$. melaninogenicus have been encountered that could grow only on enriched media in the presence of a contaminant Staphylococcus aureus colony (Holbrook, 1976). Other possible explanations are that these strains were very sensitive to exposure to oxygen, or that the colonies became pigmented as the bacterial cells died.

Bacterial antagonism may be an important factor in preventing growth of $B$. melaninogenicus. The addition of kanamycin or, more effectively, kanamycin plus vancomycin to the medium greatly reduced the number of contaminants and produced a highly significant increase in the isolation rate of B. melaninogenicus. Loesche, Hockett and Syed (1971) have previously found kanamycin to be helpful in the isolation of $B$. melaninogenicus from samples of dental plaque. Several fusobacteria are sensitive to relatively small amounts of kanamycin (Duerden et al., 1976) and addition of this antibiotic would therefore not allow an increased yield of all Gram-negative anaerobes.

The quantitative recovery of $B$. melaninogenicus was not attempted, but a clear indication of the value of VMGII transport medium and of the addition of kanamycin and vancomycin to culture media was gained and should increase the reliability of methods for the isolation of this organism.

\section{SUMMARY}

Isolation procedures involving the direct plating of specimens and the use of selective, non-selective, transport and enrichment media were compared in respect of their value for the recovery of Bacteroides melaninogenicus from the gingival sulcus. A selective medium containing kanamycin and vancomycin enhanced the recovery rate of $B$. melaninogenicus. VMGII transport medium was convenient to use and gave as good a recovery rate as that obtained with plates directly inoculated in the clinic.

We wish to thank Professor J. G. Collee and Mrs Margaret Shotter for helpful advice throughout these investigations. We also thank Mr R. Brown and Miss C. McMillan for skilled technical assistance. Financial support from the University of Edinburgh Faculty of Medicine Scholarship Fund (to W. P. H.) and from the Medical Research Council (M.R.C. Grant No. G974/325B) is gratefully acknowledged.

\section{REFERENCES}

Armitage, P. 1971. Statistical methods in medical research, Oxford, ch. 4.

BERG, J. O. AND NoRD, C. E. 1973. A method for isolation of anaerobic bacteria from endodontic specimens. Scand.J. dent. Res., 81, 163. 
Collee, J. G., Watt, B., Fowler, E. B. And Brown, R. 1972. An evaluation of the Gas-Pak system in the culture of anaerobic bacteria. J. appl. Bact., 35, 71.

Duerden, B. I., Holbrook, W. P., Collee, J. G. AND Watt, B. 1976. The characterization of clinically important Gram-negative anaerobic bacilli by conventional bacteriological tests. J. appl. Bact., 40, 163.

Finegold, S. M., Sugihara, P. T. AND SutTer, V. L. 1971. Use of selective media for isolation of anaerobes from humans. In Isolation of anaerobes, edited by D. S. Shapton and R. G. Board, London, p. 99.

HolBROOK, W. P. 1976. The isolation, culture and characterisation of Gram-negative nonsporing anaerobic bacilli with special reference to the occurrence of bacteroides species in the human mouth. Ph.D. Thesis, University of Edinburgh.

LOESCHE, W. J., HocketT, R. AND SYED, S. A. 1971. Evaluation of kanamycin as an aid in the isolation of Bacteroides melaninogenicus from dental plaque. Archs oral Biol., 16, 813.

MACPheE, I. T. AND COWLey, G. C. 1975. Essentials of periodontology and periodontics, 2nd ed., Oxford, p. 169.

MölLER, A. J. R. 1966. Microbiological examination of root canals and periapical tissues of human teeth: methodological studies. Odont. Tid., 74, Suppl., p. 365.

Ogston, S. A. 1976. Analysis of ordered trinomial data. M.Sc. Thesis, University of Edinburgh.

Rosenblatt, J. E., Fallon, A. And Finegold, S. M. 1973. Comparison of methods for isolation of anaerobic bacteria from clinical specimens. Appl. Microbiol., 25, 77.

Snedecor, G. W. And Cochran, W. G. 1967. Statistical methods, 6th ed., Ames, Iowa, ch. 12.

StARR, S. E. 1974. Comparison of isolation techniques for anaerobic bacteria. In Anaerobic bacteria: role in disease, edited by A. Balows, R. M. De Haan, V. R. Dowell JR and L. B. Guze, Springfield, Illinois, p. 47.

Sutter, V. L., Attebery, H. R., Rosenblatt, J. E., Bricknell, K. S. And Finegold, S. M. 1972. Anaerobic bacteriology manual, 1st ed., Los Angeles, p. 52.

Watt, B., Collee, J. G. AND Brown, R. 1974. The isolation of strict anaerobes: the use of an anaerobic cabinet compared with a conventional procedure. J. med. Microbiol., 7, 315.

WATt, B., HoARe, M. V. AND Collee, J. G. 1973. Some variables affecting the recovery of anaerobic bacteria: a quantitative study. J. gen. Microbiol., 77, 447.

Williams, R. A. D., Bowden, G. H., Hardie, J. M. and Shah, H. 1975. Biochemical properties of Bacteroides melaninogenicus subspecies. Int. J. syst. Bact., 25, 298. 